\title{
Ce que l'on fait dire au silence : posture, ethos, image d'auteur
}

How to make silence talk... Posture, ethos, author's image

Jérôme Meizoz

\section{OpenEdition}

12 Journals

Édition électronique

URL : http://journals.openedition.org/aad/667

DOI : $10.4000 /$ aad. 667

ISSN : 1565-8961

Éditeur

Université de Tel-Aviv

Référence électronique

Jérôme Meizoz, «Ce que l'on fait dire au silence : posture, ethos, image d'auteur », Argumentation et Analyse du Discours [En ligne], 3 | 2009, mis en ligne le 15 octobre 2009, consulté le 23 septembre 2019. URL : http://journals.openedition.org/aad/667 ; DOI : 10.4000/aad.667

Ce document a été généré automatiquement le 23 septembre 2019.

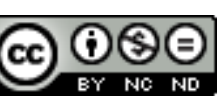

Argumentation \& analyse du discours est mis à disposition selon les termes de la licence Creative Commons Attribution - Pas d'Utilisation Commerciale - Pas de Modification 4.0 International. 


\title{
Ce que l'on fait dire au silence : posture, ethos, image d'auteur ${ }^{1}$
}

\author{
How to make silence talk... Posture, ethos, author's image
}

\author{
Jérôme Meizoz
}

[...] l'imaginaire est pris en charge par plusieurs
masques (personae), échelonnés selon la
profondeur de la scène (et cependant personne derrière).

(Barthes 1975 : 123)

1 En appendice tardif à Postures littéraires. Mises en scène modernes de l'auteur (2007), et pour donner suite à de fructueux échanges de vues avec Ruth Amossy et Dominique Maingueneau sur la pertinence et l'extension de la notion de "posture ", cet article propose une discussion de quelques termes voisins, auxquels sont confrontés tant les analystes du discours que les sociologues de la littérature. Leur articulation mérite d'être explicitée et discutée. Je le fais sans grande ambition théorique, ayant la coutume de "penser par cas", comme le préconisent Revel et Passeron (2005), quitte à reformuler ces ébauches de théorisations au contact de nouveaux faits discursifs. Que l'on prenne donc ce parcours comme une invitation à la discussion, sans en durcir les assertions.

2 Sur un plan méthodologique, la notion de "posture» m'a semblé précieuse pour dépasser la vieille division des tâches entre spécialistes de l'interne et de l'externe textuel: ainsi une posture d'auteur implique relationnellement des faits discursifs et des conduites de vie dans le champ littéraire ${ }^{2}$. Les études de cas de Postures littéraires tentent ainsi d'articuler les dimensions rhétoriques et sociologiques, à savoir le discours des acteurs et leurs prises de positions dans le champ littéraire. On y observe des conduites énonciatives et institutionnelles complexes, par lesquelles une voix et une figure se font reconnaître comme singulières dans un état du champ littéraire.

3 L'enjeu de l'articulation entre rhétorique et sociologie me semble important dans les études littéraires actuelles, au moment où de toutes parts la notion problématique 
d'« auteur » fait retour, reformulée et réévaluée à la lumière des acquis récents de plusieurs disciplines.

\section{Des notions à articuler}

4 J'ai défini la posture comme la présentation de soi d'un écrivain, tant dans sa gestion du discours que dans ses conduites littéraires publiques. Le meilleur équivalent à cette notion serait le terme latin de persona qui désigne le masque de théâtre: étymologiquement,ce à travers quoi l'on parle (per-sonare) instituant tout à la fois une voix et son lieu social d'intelligibilité. Sur la scène d'énonciation de la littérature, l'écrivain se présente et s'exprime muni de la médiation que constitue sa persona, que l'on peut appeler sa posture ou son «masque d'autorité » (Calame 2005).Ou pour le dire plus clairement: une personne n'existe comme écrivain qu'à travers le prisme d'une posture, historiquement construite et référée à l'ensemble des positions du champ littéraire ${ }^{3}$. Une posture relève d'une "scénographie auctoriale» d'ensemble. Par ce terme, Maingueneau décrit la dramaturgie inhérente à toute prise de parole, centrant son attention sur l'énonciateur du discours. Parler de posture suppose de décrire ce processus à plusieurs niveaux simultanément.

5 Ainsi, une posture n'est pas seulement une construction auctoriale, ni une pure émanation du texte, ni une simple inférence d'un lecteur. Elle relève d'un processus interactif: elle est co-construite, à la fois dans le texte et hors de lui, par l'écrivain, les divers médiateurs qui la donnent à lire (journalistes, critiques, biographes, etc.) et les publics. Image collective, elle commence chez l'éditeur avant même la publication, cette première mise en forme $d u$ discours. On la suivra dans toute la périphérie $d u$ texte, $d u$ péritexte (présentation du livre, notice biographique, photo) à l'épitexte(entretiens avec l'auteur, lettres à d'autres écrivains, journal littéraire). La posture se forge ainsi dans l'interaction de l'auteur avec les médiateurs et les publics, anticipant ou réagissant à leurs jugements.

6 J'ai étudié la posture avant tout dans les genres factuels, comme l'autobiographie, où l'auteur et l'instance de gestion du discours sont assimilés par un désormais célèbre pacte (Lejeune 1975). Mais dès que nous parlons de genres fictionnels, un nouveau problème surgit. Dans une fiction, où situer l'auteur? Quels liens faire entre l'auteur, le narrateur et les personnages? L'ethos de chacun n'a souvent rien de commun. Peut-on dès lors parler de " posture » dans une fiction?

7 Une partie du problème réside dans la confusion inhérente à la notion d'auteur et à ses usages courants ${ }^{4}$. Selon Maingueneau (2004), qui me semble en donner la meilleure description à ce jour, trois instances s'y imbriquent comme dans un anneau de Moebius :

- la personne (l'être civil);

- l'écrivain (la fonction-auteur dans le champ littéraire);

- l'inscripteur (l'énonciateur textuel).

8 En l'occurrence, étudier une posture, c'est aborder ensemble (et croiser ces données, avec la prudence requise) les conduites de l'écrivain, l'ethos de l'inscripteur et les actes de la personne. Par exemple, comprendre la relation complexe entre Michel (narrateur du roman Plateforme [2001]), Michel Houellebecq (l'écrivain en public) et Michel Thomas, le citoyen français qui se fait appeler, en littérature, Houellebecq. Il en est de même de la relation entre Ferdinand (narrateur de Mort à crédit [1936]), Louis- 
Ferdinand Céline (l'écrivain en public) et Louis Destouches, la personne civile. Lorsque Jean-Jacques Rousseau, dans les Dialogues (Rousseau juge de Jean-Jacques), dédouble son énonciation en la voix de Jean-Jacques et celle de Rousseau, il a pour visée rhétorique de dissocier deux faces de l'auteur: cette opération devait permettre de racheter l'écrivain(objet de dangereuses attaques) par les qualités de la personne. En effet, la pureté d'intentions de Jean-Jacques serait masquée au public par la célébrité de l'écrivain Rousseau. La sincérité de «Jean-Jacques » vient contrebalancer ici les risques liés au statut d'écrivain, toujours soupçonnable d'être un déguisement. En effet, devenu un personnage public, l'« écrivain" ne s'appartient plus totalement, en tant que représentation de soi, mais se connaît alors par l'image en retour que le public lui renvoie. En 1910, Lanson notait déjà : « La vie et le caractère de Rousseau ne comptent plus par ce qu'ils ont été réellement, mais par les images seules, vraies ou fausses, que les lecteurs s'en faisaient, et qui pouvaient se mêler plus ou moins aux impressions du livre » (Lanson 1910, Charles 1993 : 496).

Lorsque Rousseau rentre à Paris après un long exil, en 1770, la Correspondance littéraire témoigne d'une scène emblématique :

Il s'est montré plusieurs fois au café de la Régence, sur la place du Palais Royal ; sa présence y a attiré une foule prodigieuse, et la populace s'est même attroupée sur la place pour le voir passer. On demandait à la moitié de cette populace ce qu'elle faisait là ; elle répondait que c'était pour voir Jean-Jacques. On lui demandait ce que c'était que Jean-Jacques; elle répondait qu'elle n'en savait rien, mais qu'il allait passer 5 .

Genette radicalise cette idée, dans le cas de Stendhal, allant jusqu'à faire de la personne civile une reconstruction de l'écrivain pseudonyme: «Beyle n'est légitimement pour nous qu'un personnage de Stendhal " (Genette 1969).

11 Les actes de la personne civile ne sont donc pas exclus de l'analyse, pour autant qu'on puisse faire le lien entre eux et les conduites de l'écrivain dans le champ littéraire. Si lien il y a, c'est sans doute par l'habitus, comme ensemble des dispositions sociales d'un individu, actualisables de manière différentielle selon le champ où il est engagé. Mais je n'en suis que là pour l'instant, et pas plus loin.

\section{Ethos et posture}

12 Lors d'un séminaire international d'Analyse du discours tenu à l'EHESS en novembre 2007, à l'invitation de nos collègues norvégiens, Dominique Maingueneau s'est demandé si le terme de posture ne faisait pas double emploi avec celui d'ethos. La question est pertinente, et selon le principe du rasoir d'Ockham, on peut douter qu'il soit utile à la connaissance de multiplier les entités nominales à l'infini. Examen fait de la question, j'y réponds par la négative, dans la mesure où dans les corpus de littérature écrite, l'ethos est inféré de l'intérieur d'un discours et ne peut inclure une conduite sociale comme le vêtement porté par celui qui se présente comme écrivain : en effet, comme l'écrit Maingueneau lui-même, «l'ethos est une notion discursive, il se construit à travers le discours, ce n'est pas une "image" du locuteur extérieure à la parole » (2004: 205).

13 Pour illustrer en quoi la notion de "posture » désigne un phénomène plus large que celle d'ethos, je donne l'exemple dans mon livre, de la fameuse blouse blanche de médecin avec laquelle L.-F. Céline s'est présenté à la presse lors de la sortie de Voyage au 
bout de la nuit en automne 1932. Elément vestimentaire, externe au discours du roman, elle constitue néanmoins pour les journalistes et les lecteurs un élément à verser à leur portrait du nouvel auteur « Céline». A cela s'ajoute que le système narratif des romans céliniens invite (non sans ruse) à une lecture biographique, notamment dans Mort à crédit puis dans les récits autofictionnels comme D'un château l'autre (1957). Mon hypothèse consiste à dire que l'ethos discursif construit par Céline dans ses romans exerce une contrainte sur la mise en scène de l'écrivain en public. De même, je me suis demandé dans Le Gueux philosophe (2003) si l'ethos discursif mis en place par Rousseau dans ses deux fameux Discours $(1750,1755)$ ne pesait pas de tout son poids sur les conduites publiques que l'écrivain s'est senti tenu d'assumer jusqu'au bout. Autrement dit, l'image discursive de l'auteur construite dans le discours, s'imposant par la circulation des écrits, tend à devenir, dans ces cas, un patron de la conduite publique de l'écrivain: Céline finit par suivre le "clairon » qu'il a lui-même embouché, selon l'image très parlante de Gracq. C'est là une piste de recherche que Maingueneau trouve féconde (2004 : 206).

Pour préciser au mieux l'extension des notions d'ethos et de posture, Maingueneau m'a suggéré ceci, qui reprend la terminologie de ses travaux : l'ethos désignerait l'image de l'inscripteur donnée dans un texte singulier et pouvant se limiter à celui-ci : l'ethos des Châtiments (1853) n'est pas celui de L'art d'être grand-père (1877). De son côté, la posture référerait à l'image de l'écrivain formée au cours d'une série d'œuvres signées de son nom $^{6}$. Je trouve cette formulation très pertinente, car elle rappelle que l'ethos s'origine sur le versant discursif, alors que la posture naît d'une sociologie des conduites ${ }^{7}$. En effet, la « posture » dit la manière dont un auteur se positionne singulièrement, vis à vis du champ littéraire, dans l'élaboration de son œuvre. Une telle présentation de soi s'élabore dans la durée et de manière en quelque sorte cumulative. Entre 1762 et 1778, soit entre les Lettres à Malesherbes, les Confessions et les Rêveries du promeneur solitaire, les écrits de Rousseau manifestent plusieurs ethè distincts : de par l'ethos stoïcien qu'elles arborent (divers intertextes ont été identifiés), les Rêveries prennent le contrepied de l'image d'un auteur inquiet, ébranlé par les attaques et pressé de se justifier qui émane des Dialogues. Cependant, l'ensemble des écrits de cette dernière partie de sa vie, postérieure à l'exil et à la condamnation de ses ouvrages, contribue à une posture qui met en cohérence le discours (les écrits cités) et les conduites littéraires publiques de Rousseau (la retraite méditative, l'habit arménien). Posture d'ailleurs parfaitement identifiée, voire parodiée par les contemporains : « Singe de Diogène !», écrira Voltaire. Signifiante et promue à la célébrité dans les ultimes écrits autobiographiques de Rousseau, cette posture tire sa cohérence du fait qu'elle répond à un projet pragmatique commun à tous ceux-ci : parvenir par diverses stratégies à déjouer le complot de "ténèbres » qui l'environne. Autre exemple: dans le chapitre six de mon livre (2007: 101-109), je fais ainsi l'hypothèse qu'au cours de la trajectoire littéraire de L.-F. Céline, une même posture du "découvreur maudit» se perpétue au gré de variations d'ethos, relatives aux genres pratiqués et aux moments du champ littéraire (les romans d'avant-guerre 1932-1936; les pamphlets 1937-1941; les autofictions d'après-guerre 1944-1961). 


\section{Posture et image d'auteur}

laquelle recourt notamment Ruth Amossy dans ses travaux. Ceux-ci, comme la brillante analyse des Bienveillantes (2006) de Jonathan Littell proposée lors de la journée d'étude mentionnée précédemment, ont pour mérite de porter aussi bien sur des textes factuels que fictionnels. Du fait même de son énonciation, note Amossy, l'instance auctoriale projette une image d'elle-même dans le texte. Le lecteur se fait une image de l'inscripteur à l'aide des traces diverses laissées par celui-ci. L'image d'auteur se distingue de celle du narrateur, qui, quant à lui, est inclus dans l'univers de la fiction. En somme, l'ethos discursif de l'inscripteur constitue, il me semble, un des éléments de l'image d'auteur, car celle-ci est tissée de plusieurs types d'informations, intra- et extratextuelles.

La notion d'image d'auteur rejoint celle d'« auteur implicite » (implied author) proposée en 1961 par Wayne C. Booth dans The Rhetoric of Fiction. Dans une fine discussion de cette notion, Tom Kindt (2007) en précise les usages actuels et en fait voir tout l'intérêt pour des recherches comme les nôtres. L'idée de Booth est que se manifeste dans le texte une figure implicite d'auteur, distincte de l'auteur empirique et du narrateur fictionnel. La tripartition de Maingueneau (personne, écrivain, inscripteur) a la même vertu heuristique. Chez Booth, plus qu'une figure intentionnellement élaborée, l'auteur implicite constitue une inférence du lecteur. Dans la phraséologie de son temps, Barthes disait qu'il nous faut postuler ou désirer l'auteur pour continuer notre lecture : «Comme institution, l'auteur est mort [...] mais dans le texte, d'une certaine façon, je désire l'auteur : j'ai besoin de sa figure (qui n'est ni sa représentation, ni sa projection), comme il a besoin de la mienne (sauf à "babiller") » (1973: 45-46) l'auteur du texte qu'il lit, que ce soit par les faits discursifs ou par des connaissances biographiques, antérieures à la lecture (pour la rhétorique, autrement dit, par une saisie de son ethos préalable). En effet, chaque lecteur possède ou non des informations sur l'écrivain voire sur la personne civile, qu'il peut croiser à loisir avec les données textuelles dans son portrait de l'auteur implicite ${ }^{9}$. Alain Viala a montré également qu'une "image " s'élabore dans une série de textes attribués à la même fonctionauteur. Cette image contribue à situer l'auteur dans le champ littéraire et formate ou durcit son horizon de réception :

En donnant une œuvre, il [l'auteur] construit une image de lui-même, et, au fil des œuvres suivantes, cette image se confirme ou évolue : on attend de Gide qu'il « fasse du Gide» et qu'en même temps il soit ni tout à fait un autre, ni exactement identique au fil de ses livres (et idem pour tous) (Viala, Molinié \& Viala 1993 : 197-198).

Divers travaux ont porté aussi sur le stock d'informations (notamment biographiques) dont dispose le lecteur pour inférer. Kindt (2007) en cite principalement deux :

[David] Darby par exemple observe dans l'essai déjà mentionné sur l'histoire de la théorie narrative : « en soi, un auteur implicite est le produit de négociations entre les royaumes intratextuels et extratextuels ». Sandra Heinen s'est encore éloignée davantage de la conception selon laquelle il convient de définir l'auteur implicite uniquement comme une inférence reposant sur un texte donné. Dans un article paru en 2002 dans la revue Sprachkunst, elle propose de comprendre la notion d'auteur implicite comme l'«image» d'un auteur que se font les récepteurs

Argumentation et Analyse du Discours, 3 | 2009 
lorsqu'ils lisent un ou plusieurs de ses textes en tenant compte de toutes les

informations disponibles à son sujet (p. 74).

19 Assurément, par exemple, en lisant un roman de Michel Houellebecq précédé d'une intense rumeur médiatique, nous ne pouvons faire abstraction de toutes ces informations quand nous nous plongeons dans les propos d'un narrateur qui, dans Plateforme (2001), se nomme Michel, comme l'écrivain. Ce qui ne signifie pas que nous renoncions à la convention romanesque pour identifier purement et simplement auteur et narrateur (Meizoz 2004). Nos inférences de lecture sont cependant modifiées par ces savoirs externes ${ }^{10}$. C'est d'ailleurs, selon moi, ce que cherchent à provoquer Houellebecq ou Christine Angot, et qui alimente des débats houleux à leur sujet. Comme je l'ai montré ailleurs (Meizoz 2004), ces auteurs incluent désormais à l'espace de l'œuvre, conformément aux propositions de l'art contemporain, la performance publique d'écrivain (entretiens, lecture, etc. ${ }^{11}$. Du coup, le lecteur est invité à confronter l'inscripteur et l'écrivain, dont ces auteurs 'ambiguïsent' les rapports. Pour les littéraires qui ont comme dogme heuristique de distinguer l'auteur et le narrateur, le geste de Houellebecq, qui reprend à son compte (d'écrivain) sur un plateau de télévision les propos islamophobes de son narrateur, constitue un choc dont il faut pourtant rendre compte ${ }^{12}$.

C'est ici que l'image d'auteur peut s'articuler avec la notion de posture, qu'elle rejoint par une direction opposée. Je résumerai ainsi la division des tâches inhérentes, me semble-t-il, à ces notions :

21 - Issu d'une analyse interne, la notion d'ethos discursif concerne le discours de l'inscripteur.

- Issue d'une analyse interne, la notion d'image d'auteur concerne le discours de l'inscripteur relationnellement aux informations dont le lecteur dispose sur l'écrivain.

- Récusant la distinction de l'interne et de l'externe, la notion de posture concerne les conduites de l'écrivain relationnellement au discours de l'inscripteur et aux actes de la personne. ${ }^{13}$

22 Nous aurions donc une série de notions liées par inclusion, de la plus englobante (et donc, j'en conviens, problématique) à la plus fine :

- Posture

- Image d'auteur

- Ethos discursif

Parmi les trois scènes où se joue l'émergence du discours littéraire (Maingueneau 2004), la « scénographie » (ou scène d'énonciation) engage elle-même ces trois niveaux :

- Scène englobante

- Scène générique

- Scénographie

- - Posture

$\circ$ - Image d'auteur

- Ethos discursif

\section{Le silence signifie-t-il quelque chose?}

Pour illustrer cette discussion, je terminerai par un bref commentaire d'une célèbre nouvelle de fiction, Le silence de la mer. Pour ce faire, je croiserai les outils de lecture par 
l'ethos (Amossy 1999 et Maingueneau 2004), les savoirs historiques à disposition et une proposition de méthode formulée par Roger Chartier: "L'historicité première d'un texte est celle qui lui vient des négociations nouées entre l'ordre du discours qui gouverne son écriture, son genre, son statut, et les conditions matérielles de sa publication» (2008: 51). - Paru clandestinement le 20 février 1942 sous la signature de Vercors, ce petit volume constitue l'acte fondateur des éditions de Minuit (Paris). Il marque la naissance d'un écrivain (Vercors) que les circonstances et la fonction distinguent de la personne de Jean Bruller (1902-1991), illustrateur de presse n'ayant jamais publié d'œuvre littéraire jusque-là. A celle d'auteur pseudonyme, Bruller joint la fonction d'éditeur pseudonyme (sous le nom de Desvignes) puisqu'il est co-fondateur et animateur clandestin des éditions de Minuit: derrière la posture d'auteur, il faudrait encore envisager une posture d'éditeur, macro-figure d'énonciation qui par le choix de textes politiquement impliqués engage ici l'ensemble d'un catalogue. Mais j'en viens à l'essentiel pour nous, qu'une polémique, comme souvent, a fait apparaître: la publication du Silence de la mer suscita une querelle mémorable à sa réception, opposant notamment la lecture des résistants gaullistes et des communistes, épisode désormais retracé en détails (Simonin 1994). Pour comprendre ce conflit, quelques mots sur le récit sont indispensables.

L'intrigue est quasi contemporaine de la publication, puisqu'elle évoque l'invasion allemande. Vercors a témoigné plus tard du substrat biographique de son récit, transposé ensuite en fiction: lui-même et son épouse ont dû héberger des soldats allemands après la défaite de juin 1940. Résumons la fable : le narrateur, un homme âgé vivant avec sa nièce, doit accueillir chez lui, par ordre militaire, un officier allemand, Walter von Ebrennac, qui se révèle un homme épris de culture française. Entre les hôtes et l'invité se tisse une relation marquée avant tout par le silence, dans laquelle s'esquisse toutefois un sentiment amoureux possible entre la nièce et l'officier. Prenant conscience des intentions destructrices des Nazis à l'égard de la France, après avoir été dupé par des discours lénifiants, l'officier déçu se fait muter en première ligne, et son départ s'apparente à une forme de suicide.

L'ethos discursif du narrateur donne une image de réserve sereine, méfiante sans être agressive: disons qu'il reste sur son "quant à soi». A aucun moment, il ne juge l'officier allemand, ni n'exprime sa colère. A sa réception, en février 1942, les lecteurs de cette nouvelle, qui circula principalement dans le milieu littéraire et parmi les résistants communistes et gaullistes, inférèrent de cet ethos une image d'auteurcontrastée et problématique. Et pour cause: du fait de la clandestinité de l'édition, la plupart des lecteurs ignoraient tout à fait l'identité civile de Bruller. Celuici était avant guerre un illustrateur dont les positions de gauche modérée se rapprochèrent du gaullisme dès juin 1940. Cette ignorance de l'identité de l'auteur, qui dura jusqu'en août 1944 (Sapiro 1999 : 503), permet d'ailleurs un test en double aveugle, car l'inférence ne peut porter, ici, sur une connaissance préalable de l'écrivain. Dans ce livre, Vercors apparut ainsi à ses lecteurs tantôt comme un chantre de la résistance pacifique (c'est la lecture gaulliste), tantôt comme un traître qui, par la passivité qu'il prône face à l'ennemi, fait le jeu des ennemis de la France (c'est la lecture communiste d'Ilya Ehrenbourg, par exemple). Après d'âpres débats par presse interposée, et diverses tentatives pour discréditer la nouvelle, c'est la première image d'auteur qui triompha à la Libération, appuyée par les réseaux gaullistes de Londres qui résolurent même de faire parachuter le texte au dessus de la France. 
Mais cette réception ambiguë laissa des traces douloureuses pour Vercors, qui ressentit le besoin de corriger son texte et d'ajouter une phrase condamnant plus explicitement l'officier allemand. Dans l'édition « définitive » (selon ses vœux) de la nouvelle en 1951, on lit donc ceci : "Ainsi, il se soumet. Voilà donc tout ce qu'ils savent faire. Ils se soumettent tous, même cet homme-là. » (Vercors, Simonin 1994 : 98).

Un ethos donc, d'abord, puis une image d'auteur, produit de luttes d'interprétation, enfin une posture (d'auteur, et au-delà, d'éditeur), signalée dès le pseudonyme de l'écrivain puis manifeste dans cet ajout ou repentir tardif, ultime retouche à la nouvelle au moment où les institutions allaient en faire le monument de l'histoire littéraire pacifiée que l'on sait.

\section{BIBLIOGRAPHIE}

Amossy, Ruth (dir.). 1999. Images de soi dans le discours. La construction de l'ethos (Lausanne-Paris : Delachaux \& Niestlé)

Baroni, Raphaël (à paraître en 2009). «Ce que l'auteur fait au lecteur (que son texte ne fait pas tout seul) », L'Euvre du temps (Paris : Seuil)

Baroni, Raphaël. 2007. « Revenances de l'auteur... », Kaenel Philippe, Jérôme Meizoz, François Rosset, Nelly Valsangiacomo (dir.) La "vie et l'œuvre" ? Recherches sur le biographique, colloque tenu à l'Université de Lausanne en novembre 2007, [en ligne sur http://doc.rero.ch/record/8828?ln=fr (consulté le 18 septembre 2008)]

Barthes, Roland. 1973. Le plaisir du texte (Paris : Seuil, rééd. coll. « Points »)

Barthes, Roland. 1075. Roland Barthes par Roland Barthes (Paris : Seuil)

Bonnet, Jean-Claude. 1998. Naissance du Panthéon (Paris : Fayard)

Booth, Wayne C., 1983 [1961]. The Rhetoric of Fiction (Chicago \& New York : University of Chicago Press)

Calame, Claude. 2005. Masques d'autorité. Fiction et pragmatique dans la poétique grecque antique (Paris : Les Belles Lettres)

Célis, Raphaël. 2007. « De quelques enjeux éthiques et anthropologiques de l'oeuvre romanesque de Michel Houellebecq ", Quinche, Florence \& Antonio Rodriguez (dir.). Quelle éthique pour la littérature? Pratiques et déontologies (Genève : Labor \& Fides), p. 95-124

Charles, Michel. 1993. « Amateurs, savants et professeurs », Poétique 96, p. 493-498.

Chartier, Roger. 2008. Ecouter les morts avec les yeux (Paris : Fayard)

Delormas,Pascale. 2008. «L'image de soi dans les autographies de Rousseau », Argumentation et analyse du discours, $\mathrm{n}^{\circ} 1$, [disponible sur http://aad.revues.org/index311.html].

Darby, David. 2001. « Form and Context: An Essay in the History of Narratology », Poetics Today 22, 2001, p. 829-852 
Diaz, José-Luis. 2007. L'Ecrivain imaginaire. Scénographies auctoriales à l'époque romantique (Paris : Champion)

Genette, Gérard. 1969. «"Stendhal” », Figures II (Paris : Seuil)

Gill, Brian. 1996. «Structures d'auteur, métaphores de lecteur », Sirvent, Angeles, Josefina Bueno, Silvia Caporale (dir.). Autor y texto : fragmentos de una presencia, (Barcelona : PPU), p. 259-266

Heinen, Sandra. 2002. « Das Bild des Autors. Überlegungen zum Begriff des "impliziten Autors" und seines Potentials zur kulturwissenschaftlichen Beschreibung von inszenierter Autorschaft », Sprachkunst 33, p. 329-345

Heinich, Nathalie. 2005. L'élite artiste (Paris : Gallimard)

Kindt, Tom.2007. «L"'auteur implicite ". Remarques à propos de l'évolution de la critique d'une notion entre narratologie et théorie de l'interprétation ", Pier, John (éd.), Théorie du récit. L'apport de la recherche allemande (Lille : Presses universitaires du Septentrion) édition en ligne http:// www.vox-poetica.com/t/kindt.html, consultée le 17 septembre 2008.

Lanson, Gustave, 1965. « La méthode en histoire littéraire », Revue du mois, octobre 1910, repris, Essais de méthode (Paris : Hachette)

Meizoz, Jérôme. 2004. L'œil sociologue et la littérature (Genève-Paris : Slatkine Erudition)

Meizoz, Jérôme, 2007. Postures littéraires. Mises en scène modernes de l'auteur(Genève-Paris : Slatkine Erudition)

Revel, Jacques, Passeron, Jean-Claude (dir.). 2005. Penser par cas (Paris : Editions de la MSH, «Enquête»)

Sapiro, Gisèle. 1999. La guerre des écrivains 1940-1953 (Paris : Fayard)

Simonin, Anne. 1994. Le «Devoir d’insoumission». Les éditions de Minuit 1942-1955 (Paris : IMEC)

Viala, Alain. 1993. «Sociopoétique», Viala, Alain \& Georges Molinié. Approches de la réception, (Paris : PUF)

Weber. Max, 2003. L'éthique protestante et l'esprit du capitalisme, trad. J.-P. Grossein, (Paris :

Gallimard), p. 255-317

Saint-Amand, Denis \& David Vrydaghs. 2008. « La biographie dans l'étude des groupes littéraires. Les conduites de vie zutique et surréaliste ", COnTEXTES 3, Université de Liège, [en ligne : http:// contextes.revues.org/document2302.html, consulté le 17 septembre 2008]

\section{NOTES}

1. Je remercie Raphaël Baroni de sa relecture et de ses conseils.

2. J'emprunte la notion de « conduites de vie » (Lebensführung) à Max Weber, qui en fait une des notions-phares de l'étude des sectes protestantes, mais plus généralement un concept sociologique pour décrire les comportements sociaux dans une communauté de valeurs, cf. L'éthique protestante et l'esprit du capitalisme, pp. 255-317. Pour un usage littéraire, cf. Denis SaintAmand \& David Vrydaghs, «La biographie dans l'étude des groupes littéraires. Les conduites de vie zutique et surréaliste », 2008.

3. D'innombrables jeux auctoriaux ont pour but de détourner le caractère immédiat du pacte autobiographique, ainsi dans Roland Barthes par Roland Barthes, on lit des formules comme : "Tout ceci doit être considéré comme dit par un personnage de roman - ou plutôt par plusieurs » (Barthes, 1975, p. 123). 
4. José-Luis Diaz (2007) a développé récemment la notion de «scénographie auctoriale». Présentant les divers plans de l'instance auctoriale, il recourt à une partition différente de celle de Maingueneau: l'homme de lettres inclut la personne et l'écrivain de Maingueneau; le régisseur formel du texte recoupe l'inscripteur ; enfin, la figure de l'écrivain est une construction d'ordre imaginaire, lisible dans les textes. (José-Luis Diaz, op. cit., première partie, chapitre 1.). Cette dernière notion rejoint celle d'« image d'auteur ", à la différence près que Diaz a) mêle ainsi à tort l'écrivain (rôle social) et la personne (identité civile); b) dissocie par décret l'homme de lettres et la figure de l'écrivain, et se prive d'envisager relationnellement leur interaction et ses effets. Selon moi, c'est séparer artificiellement des phénomènes liés.

5. Correspondance littéraire, 1770, citée par Jean-Claude Bonnet, Naissance du Panthéon, p. 211.

6. Courriel de Dominique Maingueneau, 27 novembre 2007.

7. A ce sujet, voir les précisions de Pascale Delormas (2008).

8. Voir également les réflexions de Brian Gill sur $S / Z$ de Barthes, "Structures d'auteur, métaphores de lecteur ", 1996: 259-266 : "Le texte paraît donc comme un lieu où l'auteur est condamné à se fixer, qu'il le veuille ou non (Balzac le voulait, Barthes résiste). Et le lecteur, pour peu qu'il lise bien, c'est-à-dire pour peu qu'il relise et récrive, est obligé, comme Barthes, de rejeter le corps de l'auteur et de le remplacer par le sien, par un anticorps. $S / Z$ est une célébration exemplaire de ce processus. Barthes ramasse toutes les traces de la culture de Balzac dans Sarrasine, les empaquète proprement dans son code culturel, puis les fustige bien (ce sont des fatrasies, etc.) ; le corps de l'auteur est exhumé, violenté, abandonné. En même temps, la culture de Barthes essaime dans son texte, vient habiter les métaphores, les commentaires, le titre, la structuration même du livre: elle occupe tout le terrain, il n'y a de place pour rien d'autre. La lecture est bien agression, substitution (de sens et de corps), victoire sur l'Autre. »

9. Notons au passage que si l'« auteur implicite " peut être, comme l'« auteur modèle » d'U. Eco, l'image personnelle que se forge chaque lecteur, la notion d' " écrivain » définie par Maingueneau suppose une construction collective et cumulative réalisée par toute une "communauté interprétative ", selon les termes de S. Fish.

10. Voir aussi Raphaël Baroni, "Revenances de l'auteur... ", in Kaenel Philippe, Meizoz Jérôme, Rosset François, Valsangiacomo Nelly (éds.), La "vie et l'œuvre"? Recherches sur le biographique, colloque tenu à l'Université de Lausanne en novembre 2007, [disponible en ligne sur http:// doc.rero.ch/record/8828?ln=fr]. Cet article sera repris sous le titre «Ce que l'auteur fait au lecteur (que son texte ne fait pas tout seul) », in L'Euvre du temps, Paris, Seuil, coll. « Poétique », à paraître en 2009. Voir aussi le chapitre «Regarder le monde en face?" qui traite de la scénographie de La Possibilité d'une île ainsi que de la posture de Houellebecq.

11. Plus récemment, Nathalie Heinich a noté un semblable phénomène, parlant du «déplacement sur la personne de l'artiste du travail de création, de stylisation et de singularisation jusqu'alors investi sur les œuvres. Le modelage de l'artiste devient non seulement un jeu, [...] mais un véritable travail [...]. », in L'élite artiste, 2005, pp. 280-281.

12. Voir par exemple R. Baroni (2007 et 2009), J. Meizoz (2004) ou encore Raphaël Célis, «De quelques enjeux éthiques et anthropologiques de l'oeuvre romanesque de Michel Houellebecq " in Quelle éthique pour la littérature? Pratiques et déontologies, 2007, pp. 95-124.

13. La posture d'auteur, plus ou moins sciemment mise en scène, ne présume pas de sa réception effective par les publics. Il y a très souvent mouvement dialectique complexe entre la posture proposée et sa réception effective. 


\section{RÉSUMÉS}

Après la parution de Postures littéraires (2007) cet article revient sur la visée méthodologique de la notion de "posture ", en la confrontant à deux notions voisines, celles d'" ethos" et d' image d'auteur ». Il propose de hiérarchiser ces trois notions dans un ensemble conceptuel. Une brève analyse du Silence de la mer (1942) de Vercors illustre cette réflexion.

After the publication of Literary postures (2007), this article revisits the methodological approach based on the notion of "posture" by comparing it with two related notions, "ethos" and "author's image". It shows how these three notions can be organized into a hierarchy inside an integrated conceptual whole. A brief analysis of Vercors' TheSilence of the Sea (1942) illustrates this proposition.

INDEX

Mots-clés : ethos, image d'auteur, posture, scénographie

Keywords : author's image, ethos, posture, scenography

\section{AUTEUR}

JÉRÔME MEIZOZ

Université de Lausanne 